\title{
Polysèmes
}

Revue d'études intertextuelles et intermédiales

\section{Violence orphique et parole poétique dans Leaves of Grass}

Éric Athenot

\section{(2) OpenEdition}

Journals

Édition électronique

URL : http://journals.openedition.org/polysemes/1654

DOI : $10.4000 /$ polysemes. 1654

ISSN : 2496-4212

Éditeur

SAIT

Édition imprimée

Date de publication : 1 janvier 2005

Pagination : 81-99

ISSN : 0999-4203

\section{Référence électronique}

Éric Athenot, "Violence orphique et parole poétique dans Leaves of Grass », Polysèmes [En ligne],

7 | 2005, mis en ligne le 10 janvier 2017, consulté le 19 avril 2019. URL : http://

journals.openedition.org/polysemes/1654; DOI : 10.4000/polysemes.1654

Ce document a été généré automatiquement le 19 avril 2019.

Polysèmes 


\title{
Violence orphique et parole poétique dans Leaves of Grass
}

\author{
Éric Athenot
}

1 «La musique fait mal [...]. La proie de la musique est le corps humain. La musique est intrusion et capture de ce corps. Elle plonge dans l'obéissance celui qu'elle tyrannise en le prenant au piège de son chant [...]. La musique capte, elle captive dans le lieu où elle résonne et où l'humanité piétine vers son rythme, elle hypnotise et fait déserter l'homme de l'exprimable. Dans l'audition les hommes sont détenus » (Quignard 218-220). Lorsqu'il écoute, « l'auditeur reste bouche fermée : il ouvre les oreilles » (Quignard 129). C'est ainsi, fidèle à la vision énoncée par Platon au livre III de la République ${ }^{1}$, que Pascal Quignard relève le pouvoir qu'exerce le mélos sur la volonté humaine et sur le logos ainsi que le dilemme qu'il impose à l'homme, sommé de choisir entre parole et silence, libre arbitre et soumission, jouissance auditive et puissance expressive.

2 La tradition antique, de Marsyas à Orphée, accumule les récits associant la musique au meurtre brutal, l'audition à la dépossession de soi, le chant à la perte irrémédiable. Le personnage d'Orphée, tel que le dépeint Ovide aux livres X et XI des Métamorphoses ${ }^{2}$, résume à lui seul ce qui fait la dangereuse et irrésistible spécificité de son art. Fils d'Apollon et célébrant suprême des mystères de Dionysos, il conjugue l'imperturbabilité solaire de la raison à la frénésie panique de l'ivresse enthousiaste. Il endort la douleur en chantant le deuil inconsolable et paie de son corps dépecé une totale et aveugle dévotion à son art. Le mythe d'Orphée nous enseigne que la suprématie sans partage du mélos sur le logos, du chant impalpable sur le corps n'a d'issue que tragique.

Whitman, élaborant la première mouture de son recueil poétique, rêve d'unité organique et d'harmonie :

My poems when complete should be a unity, in the same sense that the earth is, or

that the human body, [...] or that a perfect musical composition is. (Whitman 763)

Dans la préface de la première édition, le poète se désigne à plusieurs reprises par le vocable de bard tout en se rêvant à la voix « eternally curious of the harmony of things with man » (Whitman 717). 
5 La musique, le chant, l'harmonie: tropes fondamentaux d'une unité fantasmée. Pour Whitman théoricien, ils figurent l'équilibre apollinien prôné en surface par la poésie ${ }^{3}$. Sous la plume du poète, ils confèrent de façon illusoire à la parole poétique la toutepuissance dionysiaque des sons musicaux. Ce dilemme agonistique qui sous-tend toute la première moitié de l'œuvre de Whitman provient de la vision paradoxale qu'a ce dernier de l'écriture, qui s'illustre, en premier lieu, par la primauté qu'il accorde à la mélodie sur le sens, au mélos sur le logos, ce qu'il tente de définir par le concept de vocalization. Pour ce poète à la recherche de la sensation pure seule la musicalité paraît garantir à l'écriture poétique une indispensable présence physique, au-delà de toute contingence sémantique, qui lui permettrait de communiquer une émotion immédiate au lecteur. Il sera vu ici à quel point ce désir de conférer une fonction phatique (ou de contact) ${ }^{5}$ à la parole poétique parcourt le discours d'un chantre qui tente d'attirer à ses côtés les citoyens d'une nouvelle république prélapsaire.

6 Le locuteur de «Song of Myself » s'arroge ainsi des caractéristiques immanquablement orphiques dès le début de son chant. En conformité avec une version tardive du mythe, Whitman choisit de mettre en avant un itinéraire qui va de Dionysos à Apollon, ce qui dans le poème se traduit magnifiquement par une progression hyperbolique allant de la vocifération - " The sounds of the belch'd words of my voice loos'd to the eddies of the wind » ( «Song of Myself » v. 25, Whitman 30) - jusqu'à l'apothéose cosmique ultime - « The feeling of health, the full-noon trill, the song of me rising from bed and meeting the sun " ("Song of Myself » v. 29, Whitman 30) dans laquelle le poète braillard devenu alouette rejoint l'astre qui engendra le chantre de Thrace. L'issue est sublime et semble jouée d'avance. Elle paraît mettre le chant du côté de l'harmonie universelle et de l'astre du jour à son zénith.

7 Notre lecture de Leaves of Grass s'attachera toutefois à suivre le parcours orphique de la figure énonciatrice pour en souligner la dimension éminemment tragique. Explorer le rapport douloureusement passionné de la figure du locuteur whitmanien et de la musique, assister aux violences paroxystiques auxquelles le mélos soumet le corps du et dans le texte à travers l'éclatement des figures rhétoriques, creuser enfin l'exploration inquiète de l'adéquation chant orphique/parole poétique nous amènera à poser la question de la viabilité communicative d'une entreprise qui prétend donner chair au logos , qui tente d'ériger la singularité de son expression en parole universelle, qui s'acharne à vouloir figurer le réel, qui rêve de s'ouvrir à autrui tout en exhibant un Moi envahissant, et qui offre enfin au lecteur comme ultime moyen d'appropriation du texte le sacrifice expiatoire de ce corps chantant.

8 Le premier vers de "Song of Myself » identifie et isole sans complexe le Moi du poète comme objet de célébration :

I celebrate myself, and sing myself.

(« Song of Myself » v. 1, Whitman 28)

9 Parfait pentamètre iambique, ce vers place l'entreprise poétique de Whitman sous le signe de l'épopée personnelle. Le parallèle avec le début de L'Énéide a été maintes fois souligné. La différence avec Virgile est grande cependant. Les faits d'armes glorieux et les hommes illustres font place à présent au quotidien d'un humble imprimeur new-yorkais, fils du peuple anonyme d'une métropole en plein devenir. La célébration dithyrambique de la lignée d'Auguste cède ici la place au triomphe du Moi qui s'énonce à trois reprises en l'espace de ce seul vers et qui souligne la dimension tautologique de la poésie whitmanienne. Le périple qui s'annonce en préambule du chant fondateur de la poésie américaine semble parcourir d'emblée la distance qui va de I à myself révélant par là 
même l'étonnante et dangereuse facilité du sujet d'énonciation à se prendre comme unique objet de son discours. Cette tentation du solipsisme s'accompagne immédiatement d'une intention représentative affichée dès les vers suivants :

And what I assume you shall assume,

For every atom belonging to me as good belongs to you.

(«Song of Myself », v. 2-3, Whitman 28)

\section{de la poésie :}

Beginning my studies the first step pleas'd me so much, The fact consciousness, these forms, the power of motion,

The least insect or animal, the senses, eyesight, love,

The first step I say awed me and pleas'd me so much,

I have hardly gone and hardly wish'd to go any farther,

But stop and loiter all the time to sing it in ecstatic songs.

(« Beginning My Studies », Whitman 9)

Lors de cet étalage impudique de jouissance physique, le lecteur assiste à la venue au monde allégorique du Moi whitmanien. Le cri primal est ici chant de l'extase et il résonne tout entier de l'ubiquité pronominale qui fait la singularité de Leaves of Grass (une ubiquité de nature ici avant tout scopique comme le souligne le redoublement du phonème dans eyesight - la vue - le seul sens mentionné de façon explicite). Jouissance de soi, célébration du Moi en parfaite adéquation avec un macrocosme tenu à distance respectable par le regard, tels semblent être les fondements heuristiques premiers de la geste whitmanienne.

Il n'est pas surprenant dans ce cas que la musique agresse la figure énonciatrice d'une violence sans pareille. Quignard souligne avec malice que l'ouïe est le sens premier qui force l'homme à percevoir le monde :

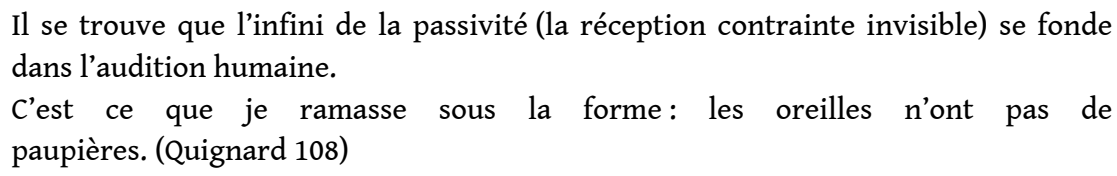

Le Moi whitmanien chante la vue, sens flatteur qu'il maîtrise à son gré et à son avantage dans son rapport au monde. La violence qui entoure la perception de la musique dans la poésie de Whitman tient à l'invasion subie par la psyché du locuteur et à la terrible passivité qu'elle engendre chez celui-ci. Quignard, à ce propos, rappelle qu'obéir vient du latin obaudire qui signifie entendre ${ }^{6}$. Cette passivité fait bien vite voler en éclat l'équanimité satisfaite d'une figure énonciatrice qui se décrit dans le poème "Me Imperturbe ", dont le titre peut se lire comme un oxymore flagrant, comme étant capable d'affronter la violence du monde avec l'indifférence des créatures des règnes végétal et animal :

[...] Me wherever my life is lived, $\mathrm{O}$ to be self-balanced for contingencies,

To confront night, storms, hunger, ridicule, accidents, rebuffs, as

the trees and animals do.

(« Me Imperturbe » v. 7-8, Whitman 11)

La musique telle que la présente la vingt-sixième section de "Song of Myself », sur laquelle nous allons nous attarder un instant, bouleverse radicalement cet équilibre fragile en mettant le locuteur dans la position précaire de récepteur sonore : 
Now I will do nothing but listen,

To accrue what I hear into this song to let sounds contribute toward it

(« Song of Myself » v. 582-583, Whitman 55)

\section{énonciatrice :}

I hear bravuras of birds, bustle of growing wheat, gossip of flames, clack of sticks

cooking my meals,

I hear the sound of love, the sound of the human voice,

I hear all sounds running together, combined, fused or following.

(« Song of Myself » v. 584-586, Whitman 55)

Les nombreuses allitérations des bilabiales initiales (bravura, birds, bustle), des explosives ( clacks, sticks, cooking) et des sifflantes (gossip, sticks, sounds) associées à l'anaphore (I hear) et à la perception anthropomorphe des sons (gossip of flames, all sounds running together) transcrivent l'enthousiasme auditif du sujet qui écoute. Le phénomène se poursuit et s'intensifie ensuite lors de la description des différentes activités humaines de Manhattan (v. 587-590).

La composition poétique opère un crescendo progressif vers un tintamarre urbain proprement assourdissant :

The heave'e'yo of stevedores unlading ships by the wharves,

The refrain of the anchor-lifters,

The ring of the alarm-bells, the cry of fire, the whirr of swift-streaking engines and

hose-carts with premonitory tinkles and color'd lights,

The steam-whistle, the solid roll of the train of approaching cars,

The slow march play'd at the head of the association marching two and two,

(They go to guard some corpse, the flag-tops are draped with black muslin.)

( Song of Myself» v. 591-595, Whitman 56)

19

Les procédés relevés plus haut sont également présents ici, mais la scène décrite frappe par la rapidité avec laquelle le locuteur passe de la célébration des interjections des marins au travail à l'incendie et à la cacophonie urbaine qui résulte de celui-ci. De la célébration de la vie et des rythmes du labeur quotidien, le poème passe par la parataxe la plus abrupte à l'accident, aux bruits stridents et à la mort. La polyphonie urbaine tant prisée du locuteur se mue en marche funèbre (slow march) et prépare le lecteur à la mort allégorique de celui-ci à la fin des quatre strophes qui suivent :

I hear the violoncello, ('tis the young man's heart's complaint,)

I hear the key'd comet, it glides quickly in through my ears,

It shakes mad-sweet pangs through my belly and breast.

I hear the chorus, it is a grand opera,

Ah this indeed is music - this suits me.

A tenor large and fresh as the creation fills me,

The orbic flex of his mouth is pouring and filling me full.

I hear the train'd soprano (what work with hers is this?)

The orchestra whirls me wider than Uranus flies,

It wrenches such ardors from me I did not know I possess'd them,

It sails me, I dab with bare feet, they are lick'd by the indolent waves,

I am cut by bitter and angry hail, I lose my breath,

Steep'd amid honey'd morphine, my windpipe throttled in fakes of death,

At length let up again to feel the puzzle of puzzles,

And that we call Being.

(« Song of Myself », v. 596-610, Whitman 56) 
passage est remarquable à plus d'un titre. Il offre un étonnant résumé de ce que nous analyserons comme la dimension tragique de la poésie whitmanienne et du rôle capital que joue la musique dans le déroulement de la parole poétique selon Whitman.

Les figures rhétoriques affichent une correspondance d'un mimétisme saisissant avec le propos: outre l'anaphore et les nombreuses allitérations, c'est avant tout l'hyperbole, trope whitmanien de la violence par excellence, qui frappe ici. Cette dernière sert à souligner les affres de la jouissance auditive dans lesquels est plongé le locuteur. Le vers 604 (The orchestra whirls me wider than Uranus flies) en offre un exemple saisissant. L'agrandissement cosmique du locuteur, outre le fait de faire référence à la musique des sphères chère au mythe d'Orphée, signifie au propre et au figuré le transport érotique auquel il est ici sujet ${ }^{7}$. L'hyperbole «rend compte d'une pensée accélérée artificiellement ", comme l'a montré Michaux avec la mescaline ${ }^{8}$. C'est ici la musique savante qui fait office de substance narcotique et qui agit sur l'auditeur comme la morphine mentionnée au vers 608. La déroute des sens du locuteur s'illustre en outre par un recours à la dérivation (filling me full), le pléonasme (It wrenches such ardors from me I did not know I possess'd them, où le dernier pronom vient en trop du reste de la phrase), l'oxymore (mad-sweet), et culmine dans l'énallage final où le $I$ cède la place de façon abrupte à un we inattendu. Ce passage fulgurant du I au we signe l'anéantissement de la singularité du locuteur et complète le travail de sape mené par le passage de la voix active (I hear) au passif (I am cut by bitter and angry hail v. 607). Entre-temps, le I aura été réduit par la syntaxe à l'état d'objet (v. 603-605) et la violence se sera faite nettement plus traumatique.

La mort finale résulte de la mise en pièces violente du locuteur et de sa disparition du discours en tant que sujet unique de perception. La mise en lambeaux de celui-ci se double dans ce passage d'un recours systématique à la métonymie. Est ici proposé à travers l'accumulation des différentes parties d'un corps tout entier violenté et mis en morceaux par l'écoute musicale (les oreilles par lequel les sons s'introduisent rapidement et inéluctablement, le ventre, la poitrine, les pieds, puis la trachée) un blason macabre du locuteur écartelé (Cf. cut, v. 607), puis finalement étouffé. Ce trépas orphique, petite mort extatique parmi tant d'autres chez Whitman, résonne d'un écho melvillien qu'introduit le mot fakes au vers 608. La note de l'édition Norton indique sommairement : «fakes: The turns or coils of a rope. » (Whitman 57). Il est aisé, dans la lecture tragique de Leaves of Grass proposée ici, de voir dans cet étouffement un parallèle poétique à la mort également paroxystique du capitaine Achab, étranglé par la corde de son harpon et ainsi réduit au silence avant de disparaître sous les flots :

[...] the flying turn caught him round the neck, and voicelessly as Turkish mutes bowstring their victim, he was shot out of the boat, ere the crew knew he was gone.

(Melville 684)

La mort dans les deux cas réduit au silence. Elle fait taire la voix et noie le sujet dans un océan de signifiants mortifères. Le champ sémantique maritime du vers 606 (Cf. sails et waves) présente la suffocation du locuteur whitmanien comme l'équivalent poétique de la noyade d'Achab au terme de sa quête autodestructrice. Cet écho peut-être fortuit ${ }^{9}$ situe sans équivoque le destin du locuteur de Leaves of Grass et la quête poétique de son créateur dans la sphère tragique dont il convient enfin de définir la nature.

La violence musicale décrite dans la vingt-sixième section de Song of Myself soulève avant tout le problème de la nature du projet poétique whitmanien dans son ensemble. Travail d'objectivation, passage de l'affect vers la perception ${ }^{10}$, de l'expérience sensuelle vers 
l'expression linguistique, il convient d'interroger l'œuvre comme odyssée allégorique du je vers le moi, puis du je vers le tu. «Song of Myself », coup d'éclat inégalé de la production whitmanienne, décrit une parabole du premier vers (I celebrate myself, and sing myself [Whitman 28]) jusqu'au vers conclusif (I stop somewhere waiting for you [Whitman 89]). Passage du chant à la stase ataraxique, de la recherche de soi à l'ouverture à l'autre. Tel semble être la visée ontologique du poète qu'il exprime en des termes empreints de rhétorique démocratique :

The messages of great poets to each man and woman are, Come to us on equal terms, Only then can you understand us, We are no better than you, What we enclose you enclose, What we enjoy you may enjoy.

(Whitman 719)

Le modal de la fin signale pourtant l'ambiguïté du propos de Whitman. S'agit-il d'une égalité accordée de facto par la poésie ou seulement d'une probabilité empathique fondée sur la perception diffuse mais inexprimée d'une différence à jamais insurmontable?

L'une des réponses apportées par la vingt-sixième section de "Song of Myself » et sa cadence mystérieuse semble aller dans le sens d'une fusion, d'une invivable absorption de l'Autre par le Moi. Ce danger est proposé comme une vertu par le poète lorsqu'il préface la première édition de Leaves of Grass :

The proof of a poet is that his country absorbs him as affectionately as he has absorbed it.

(Whitman 731)

Cette rhétorique pseudo-cannibale de l'assimilation se retrouve tout au long des poèmes et est associée à l'obsession égotiste du locuteur :

I know perfectly well my own egotism,

Know my omnivorous lines and must not write any less,

And would fetch whoever you are flush with myself.

( Song of Myself », v. 1083-1085, Whitman 77)

La jouissance égotiste exprimée sans retenue par le locuteur de «Song of Myself » peut aller jusqu'à la négation de toute différence existant entre l'Autre et le Moi :

In all people I see myself, none more and not one a barley-corn less,

And the good or bad I say of myself I say of them.

( Song of Myself » 401-402, Whitman 47)

Ontologie égalitaire face au macrocosme auquel donnerait accès la poésie de Whitman ou négation de toute différence ontologique? La violence que provoque la musique chez le locuteur du poème semble indiquer à quel point l'irruption d'un corps étranger (fût-il impalpable) dans sa psyché ${ }^{11}$ déconstruit le lien statique qui réconforte son sentiment d'illusoire ipséité12 par rapport au macrocosme. Le Moi whitmanien se trouve alors plongé dans une vulnérabilité douloureuse que ne pallie guère le recours abrupt et abusif au we conclusif relevé plus haut.

Le logos whitmanien, avide de totalité et d'ouverture cosmique, ne peut en fin de compte s'extraire de l'emprise qu'exerce sur lui le réel qu'en détournant celui-ci au profit du chant de son seul Moi :

The city sleeps and the country sleeps,

The living sleep for their time, the dead sleep for their time,

The old husband sleeps by his wife and the young husband sleeps by his wife;

And these tend inward to me, and I tend outward to them,

And such as it is to be one of these more or less I am, 
And of these one and all I weave the song of myself.

(« Song of Myself » 324-329, Whitman 44)

31 S'étale dans ces vers l'hybris tragique de la destinée whitmanienne, son incapacité fondamentale à s'ouvrir objectivement à l'Autre. Le désir de totalité s'exprime ici d'un point de vue géographique, temporel et ontologique. Le poète est victime du déni d'altérité analysé ainsi par Lévinas et rapporté par Derrida :

La « décence » et la « vie courante » nous font croire à tort que « l'autre est connu par la sympathie, comme un autre moi-même, comme l'alter ego ».

(Derrida 184)

Cette sympathie chez Whitman est à prendre dans son acception musicale, comme les cordes d'un luth ou d'un théorbe qui résonnent sous l'action des vibrations émises par celles effectivement pincées par le musicien. Le terme apparaît ainsi mentionné dans la poésie :

And nothing, not God, is greater to one than one's self is,

And whoever walks a furlong without sympathy walks to his own funeral drest in

his shroud.

(« Song of Myself », v. 1271-1272, Whitman 86)

Au-delà de ce désir d'ouverture et d'union empathique avec l'Autre, la musique semble en fin de compte mettre à jour chez Whitman l'incapacité de son discours à résister aux assauts violemment solliciteurs du réel.

La tragédie vécue par le logos whitmanien est d'autant plus complète que celui-ci semble se soustraire aux efforts déployés par le poète pour en limiter l'atomisation lorsqu'il tente de revendiquer la contradiction comme force dynamique de son discours :

Do I contradict myself?

Very well then I contradict myself,

(I am large, I contain multitudes.)

(« Song of Myself » 1324-1326, Whitman 88)

L'aveu hyperbolique exprimé entre parenthèses sonne l'irrémédiable résistance d'un médium, le logos, qui échappe à toute tentative d'appropriation de la part de son créateur ${ }^{13}$. Ce dernier vers, célèbre entre tous, est lu comme la devise d'un poète amant et chantre du monde. L'ambiguïté sémantique du verbe (rendue par l'équivalent français contenir) semble au contraire démontrer la censure effectuée par la langue poétique de Whitman, qui donne à voir le monde mais jamais à l'entendre directement, qui retient le réel autant qu'elle le montre, et qui détourne le sociolecte dans un idiolecte violemment bigarré. La crainte affichée par le discours whitmanien d'une inéluctable ouverture sémantique est soulignée ailleurs dans le poème sous forme dialogique :

Speech is the twin of my vision, it is unequal to measure itself,

It provokes me forever, it says sarcastically,

Walt you contain enough, why don't you let it out then?

(«Song of Myself », v. 566-568, Whitman 55)

La dérive aporétique qui menace le discours whitmanien est ici soulignée par la fêlure que révèle la question nullement rhétorique posée par le logos à la figure de son créateur. Comment la parole omnivore d'un seul poète pourrait-elle se prétendre la somme synchronique du langage humain dans son ensemble et survivre à ce dessein insensé mais maintes fois réitéré dans les écrits théoriques de Whitman ${ }^{14}$ ?

L'aporie sera en partie résolue, en conformité avec le mythe d'Orphée, par le meurtre du locuteur à l'origine de ce logos mégalomane : 
He most honors my style who learns under it to destroy the teacher.

(«Song of Myself», v. 1236, Whitman 84)

Ce meurtre du Moi énonciateur permet l'accès de l'Autre (ici le lecteur) à l'œuvre par-delà la figure du poète fantasmée à outrance au fil des vers. Cette mise à mort réduit à néant la fracture de l'énonciateur et du macrocosme. Cette destruction orphique répond ainsi à l'effroi tragique du chantre en pleine anagnorèse dans «As I Ebb'd with the Ocean of Life» :

I perceive I have not really understood anything, not a single object, and that no man ever can,

Nature here in sight of the sea taking advantage of me to dart upon me and sting me,

Because I have dared to open my mouth to sing at all.

(« As I Ebb'd with the Ocean of Life », v. 32-34, Whitman 254)

Le chant whitmanien est perçu ici comme contre-nature. Le Moi se désagrège sous les assauts violents de la nature et chante son chant du cygne :

(See, from my dead lips the ooze exuding at last,

See the prismatic colors glistening and rolling,)

( « As I Ebb'd with the Ocean of Life » v. 59-60, Whitman 256)

Surgit du cadavre du poète-Orphée en décomposition l'arc-en-ciel, symbole de la réconciliation entre Dieu et les hommes, figure visuelle de la richesse d'une parole qui s'achève dans l'apaisement d'une appropriation enfin possible par le lecteur ${ }^{15}$.

41 La confrontation du logos whitmanien et du mélos semble donc illustrer les efforts de communication déployés par le Moi qui s'écrit au fil des poèmes en direction de l'Autre qui lit. Fragmentée par la rencontre, déchiquetée par l'invasion du réel, la parole poétique effectue le périlleux périple de la sensation à l'objectivation, de l'expérience à l'expression ${ }^{16}$. Elle montre le travail du verbe comme le combat sans issue entre le sens et les sens, entre l'écrit et le vécu, entre langue et parole. Ce destin tragique de l'écriture réduit Whitman à tenter de créer un idiome qui lui soit propre au moyen de figures qui confèrent un semblant de virulence au logos, ce qui, selon Deleuze, motiverait chez ce poète la recherche d'une « langue convulsive $»^{17}$. La musique, force dionysiaque du désir d'immédiateté à jamais contrarié, installe au sein du discours poétique whitmanien le chaos qui malmène le discours et le contraint à se laisser enfin approprier par le lecteur, violence ultime en conformité avec ce que Lyotard écrit de la poésie radicale qui «descend jusqu'au plus bas échelon de la hiérarchie des unités linguistiques et [...] importe l'agitation, l'insurrection, non plus seulement dans la prosodie traditionnelle [...] mais dans les règles fondamentales $d u$ discours communicable luimême » (Lyotard 61-62).

La violence novatrice du logos whitmanien donne une tonalité véritablement tragique au chant de ce Moi singulier. Frappée de plein fouet par l'entropie, la fragmentation, le solipsisme et la tautologie, la parole whitmanienne soulève en dernière analyse le problème de la poésie comme outil de communication marginal. La violence qui menace de faire voler en éclat le discours whitmanien, qui ouvre celui-ci à toute entreprise de possession, celle-là même qui tente d'annihiler la figure du Moi évoluant au fil des vers, ne pourrait-elle pas être perçue comme le symptôme logocentrique d'une conscience aiguë de la marginalité ? L'ambition idéologique du poète, son désir non seulement d'intégrer mais de revivifier la norme donne lieu dans son œuvre à une illusion qui ne manque pas d'évoquer ce que Lyotard nomme l'«absolu de totalité »... C'est à cette incapacité du signe à épouser le réel que le logos de Whitman, tout autant qu'un autre, est 
confronté au fil des poèmes. Les ruses déployées pour faire accroire au lecteur qu'il trouve une issue en sacrifiant la figure énonciatrice des poèmes sont vite contredites par les poussées centrifuges des tropes :

One of the centripetal and centrifugal gang I turn and

talk like a man leaving charges before a journey.

( Song of Myself », v. 111, Whitman 79)

Devant les tiraillements inexorables de son logos, le poète dépose les armes, preuve s'il en est que sa poésie, orphique dans son éclatement ultime, peut paraître un assemblage de signifiants exogènes et parfois discordants... Elle offre, cependant, par sa tonalité tragique et son sens aigu de la perte sensorielle la seule consolation que puisse réellement apporter toute œuvre littéraire à celui qui la parcourt : celle d'offrir en abyme au lecteur l'expression d'une destinée autre et pourtant douloureusement semblable, distante dans son déroulement rhétorique mais indéniablement proche dans ses résonances intimes. Whitman par le biais du sort réservé à la figure de son Moi recréé nous enseigne que créateur et lecteur ne peuvent faire l'économie d'une violence mutuelle, phénomène que l'on désignera par ce que Badiou nomme "l'intériorisation du deux »... Au-delà de l'axiome rimbaldien - «Je est un autre »-, la musique ouvre enfin souverainement Leaves of Grass à la féconde cohabitation des deux termes indispensables à tout projet de parole et de lecture : je et un autre.

\section{BIBLIOGRAPHIE}

Bauerlein, M. Whitman and the American Idiom. Baton Rouge and London: Louisiana State UP, 1991.

Beach, C. The Politics of Distinction, Whitman and the Discourses of Nineteenth-Century America. Athens and London: The U of Georgia P, 1996.

Deleuze, Gilles. Critique et clinique. Paris : Éditions de Minuit, 1993.

Derrida, Jacques. L'Écriture et la différence. Paris : Éditions du Seuil, 1967.

Docherty, T. Alterities. Oxford: Oxford UP, 1996.

Dupriez, Bernard. Les Procédés littéraires. Paris: 10/18, 1997.

Lalande, A. Vocabulaire technique et critique de la philosophie. Paris : PUF, 1926.

Lyotard, Jean-François. Discours, Figure. Paris : Klincksieck, 1985.

Melville, Herman. Moby Dick. London: Penguin Books, 1972.

Ovide. Les Métamorphoses. Trad. Georges Lafaye. Paris : Gallimard, 1992.

Plato. The Republic. Trans. Desmond Lee. London: Penguin Books, 1955.

Quignard, Pascal. La Haine de la musique. Paris : Gallimard, 1996.

Whitman, Walt. Leaves of Grass. Ed. Sculley Bradly and Harold W. Blodgett. New York and London, W.W. Norton \& Company, 1973. 


\section{NOTES}

1. Plato, The Republic, London, Penguin, 1955, 157-161.

2. Ovide, Les Métamorphoses, Paris, Gallimard, 320-323 et 350-352.

3. Cf. l'analyse que fait Bauerlein du terme tally.

4. M. Bauerlein, Whitman and the American Idiom, Baton Rouge and London, Louisiana State University Press, 1991, 41 : «"Vocalization" and "eloquence” possess "attraction”, "magnetism", "power", and "electrical spirit", that particular emotional pull whitman luxuriated in and hoped to exercise upon others in his own projected lecture career and through his poetry. »

5. B. Dupriez, Les Procédés littéraires, Paris, 10/18, 1997, 182 : « Avec une énonciation centrée sur le contact entre les interlocuteurs, le texte a la fonction "phatique" ou "de contact" ".

6. P. Quignard, La Haine de la musique, Paris, Gallimard, 1996, 108.

7. Ibid., 119: "Pourquoi y eut-il des auditions humaines 1.collectives, 2. circulaires ou quasi circulaires? En langue grecque le cercle magique se dit orchestra. Le cercle auditif ou la ronde dansée configurent dans l'espace ce que in tempore illo inscrit l'ordre du temps. »

8. B. Dupriez, Les Procédés littéraires, op. cit., 238-239.

9. Rien n'indique en effet que Whitman ait jamais lu Moby Dick.

10. A. Lalande, Vocabulaire technique et critique de la philosophie, Paris, PUF, 1926, 701.

11. Mais voir également les $27^{\mathrm{e}}$ et $28^{\mathrm{e}}$ sections, qui décrivent les affres du contact physique dans la psyché du locuteur qui se découvre alors «a new identity ». («Song of Myself », v. 611-641, Whitman 57-58)

12. J. Derrida, L'Écriture et la différence, Paris, Éditions du Seuil, 1967, 184.

13. "English is the powerful language of resistance." W. Whitman, Leaves of Grass, op.cit., 729-730.

14. «The sailor and traveler... the anatomist, chemist, astronomer, geologist, phrenologist, spiritualist, mathematician, historian and lexicographer are not poets, but they are the lawgivers of poets and their construction underlies the structure of every perfect poem. » ibid., 720.

15. L'Apocalypse de St Jean 4:3 et 10:1.

16. Jean-François Lyotard, Discours, Figure, Paris, Klincksieck, 1985, 30.

17. Gilles Deleuze, Critique et clinique, Paris, Éditions de Minuit, 1993, 77.

\section{INDEX}

oeuvrecitee Leaves of Grass 